\title{
Glycogen Accumulation and the Induction of Nitrogenase Activity in the Heterocyst-forming Cyanobacterium Anabaena variabilis
}

\author{
By ANNELIESE ERNST AND PETER BÖGER* \\ Lehrstuhl für Physiologie und Biochemie der Pflanzen, Universität Konstanz, \\ D-7750 Konstanz, West Germany
}

(Received 4 April 1985; revised 24 June 1985)

\begin{abstract}
Anabaena variabilis (ATCC 29413) grown in batch cultures for 2 d exhibited rapid glycogen synthesis upon dilution of the cell suspension. The cellular polysaccharide concentration attained a maximum after 9-12 h growth in fresh medium and decreased thereafter. The growth rate, the rate of glycogen accumulation and the maximum amount of glycogen increased when light intensity was increased. Accumulation occurred with both $\mathrm{N}_{2}$ and ammonia as $\mathrm{N}$-sources. In cultures grown in the presence of ammonia, the increase in the cellular $\mathrm{C}$-fraction upon dilution caused neither induction of nitrogenase nor heterocyst formation. However, ammonia depletion during subsequent growth gave rise to a second accumulation of glycogen followed by differentiation. Thus, in this phototroph, glycogen is synthesized without subsequent differentiation following an increase in average irradiation as long as the $\mathrm{N}$-supply is maintained. Glycogen synthesis following $\mathrm{N}$-depletion, however, is accompanied by induction of nitrogenase.
\end{abstract}

\section{INTRODUCTION}

A polysaccharide with many of the features of a carbon and energy reserve is stored in cyanobacteria as discrete granules or rods located between the thylakoids. By staining and chemical analysis it has been shown to conșist of D-glucose monomers and to resemble glycogen in structure and branching (for reviews see Smith, 1982; Allen, 1984). An accumulation of polysaccharides in microbes usually occurs as a result of limiting growth conditions in the presence of an excess source of carbon. Cyanobacteria accumulate glycogen when growth ceases because of the depletion of a nutrient such as nitrogen (Allen \& Smith, 1969; Lehmann \& Wöber, 1976) or when grown under suboptimal temperature conditions (van Eykelenburg, 1980). However, two phototrophs, Rhodopseudomonas capsulata and Anabaena variabilis, have been shown to accumulate large amounts of glycogen during the exponential phase of growth (Eidels \& Preiss, 1970; Ernst et al., 1984). Accumulation of products of photosynthesis is observed before nitrogenase induction (Kulasooriya et al., 1972; de Vasconcelos \& Fay, 1974) and was shown to influence nitrogenase synthesis (Rippka \& Stanier, 1978) and activity (Ernst et al., 1984). In addition a periodic accumulation of carbohydrates was observed when cyanobacterial cells were grown in a nutrient-sufficient turbidostat in light-dark cycles (van Liere et al., 1979; Kromkamp \& Mur, 1984).

As pointed out by Mur (1983), little is known about the regulation of reserve material synthesis in phototrophs and the possible adaptation of these organisms to periodic variations in light energy supply during diurnal cycles. This study, for the first time, provides evidence for glycogen accumulation induced by enhanced photosynthetic activity without the interference of $\mathrm{N}$-metabolism. This accumulation differs from accumulation during $\mathrm{N}$-limitation by not being followed by differentiation in a heterocyst-forming cyanobacterium.

Abbreviation: Chl, chlorophyll. 


\section{METHODS}

Organism and growth. Anabaena variabilis (ATCC 29413) was grown in a thermostatically-controlled waterbath (Kniese-Edwards, Marburg, Germany) as described by Ernst et al. (1984). The mineral medium contained negligible amounts of combined nitrogen (Arnon et al., 1974). Cells grown in this medium fixed $\mathrm{N}_{2}$ and were regularly diluted every $2 \mathrm{~d}$. For some experiments the mineral medium was supplemented with ammonium chloride $(6 \mathrm{~mm})$ and the buffer (sodium/potassium phosphate) concentration was increased fourfold. The preadaptation of cells to ammonia was for a minimum of 16 generations. Thereafter, cultures were free of heterocysts and nitrogenase activity. As ammonia was consumed within $30 \mathrm{~h}$ (see Fig. 4), cultures were transferred to fresh medium daily to avoid $\mathrm{N}$-limitation and subsequent heterocyst induction.

Estimations. Glycogen was specifically quantified by extraction, enzymic hydrolysis and a coupled enzymic assay of glucose as described in Ernst et al. (1984); this latter paper also includes references for the determination of nitrogenase activity, $\mathrm{Chl}$ and dry weight. Ammonium concentration was measured with a double-beam spectrophotometer (Shimadzu UV-150) according to Chaney \& Marbach (1962) in culture medium cleared by centrifugation $(5 \mathrm{~min}, 5000 \mathrm{~g}$ ). Representative results from eight experimental series are shown; each data point was obtained from duplicate or triplicate determinations.

Chemicals. Enzymes and glycogen (from molluscs) were purchased from Boehringer; other compounds used were of the highest analytical grade available.

\section{RESULTS}

\section{Growth and synthesis of glycogen}

A. variabilis was cultivated with $\mathrm{N}_{2}$ as the sole nitrogen source. Cultures grown at $4 \mathrm{~W} \mathrm{~m}^{-2}$ and $12 \mathrm{~W} \mathrm{~m}^{-2}$ incident irradiation were used for inoculation after having reached a cell density equivalent to $10 \mu \mathrm{g} \mathrm{Chl} \mathrm{ml}^{-1}$ within 72 and $48 \mathrm{~h}$, respectively. Dry weight increased immediately upon inoculation, while a 1 to $2 \mathrm{~h}$ lag-phase was observed in Chl synthesis (Fig. $1 \mathrm{a}$, $b)$. Thereafter growth proceeded exponentially. Growth rates, estimated from the increase in $\mathrm{Chl}$, were $0.06 \mathrm{~h}^{-1}$ (doubling time $12 \mathrm{~h}$ ) at $4 \mathrm{~W} \mathrm{~m}^{-2}$ and $0.08 \mathrm{~h}^{-1}$ (doubling time $8.6 \mathrm{~h}$ ) at $12 \mathrm{~W} \mathrm{~m}^{-2}$. Acetylene-reducing activity (nitrogenase) increased at rates of $0.08 \mathrm{~h}^{-1}$ (Fig. $1 \mathrm{a}$ ) and $0 \cdot 10 \mathrm{~h}^{-1}$ (Fig. 1 b), respectively. In contrast, glycogen increased linearly with time at a constant rate of synthesis of $0.9 \mu \mathrm{g} \mathrm{ml}^{-1} \mathrm{~h}^{-1}$ (Fig. $1 a$ ) and $2 \cdot 3 \mu \mathrm{g} \mathrm{ml}^{-1} \mathrm{~h}^{-1}$ (Fig. $1 b$ ) during exponentialphase growth. It should be noted that net synthesis of glycogen is compared to other parameters on a logarithmic scale in Fig. 1. A linear plot is shown in Fig. 4.

Over a period of 9 to $12 \mathrm{~h}$ the relative increase of glycogen was higher than that of the other parameters measured. As a result, glycogen accumulated in the cells and attained a maximum of $23 \%$ and $35 \%$ of the total dry matter (Fig. 2 ) in the cultures characterized in Figs $1(a)$ and $1(b)$, respectively. After this initial growth phase, the net increase in glycogen became smaller than that of the other parameters and thus the glycogen content of the cells decreased. During the initial growth phase neither the rate of glycogen synthesis nor the maximum glycogen content could be enhanced by dilution of the culture at a selected light intensity (Figs $1 b$ and 2).

\section{The influence of the $N$-source}

Heterocyst-free filaments, grown with ammonia as a combined $\mathrm{N}$-source at an incident irradiation of $12 \mathrm{~W} \mathrm{~m}^{-2}$, exhibited a growth rate of $0.11 \mathrm{~h}^{-1}$ (Fig. 3). As in $\mathrm{N}_{2}$-fixing cultures, glycogen increased linearly with time (rate of synthesis: $2.4 \mu \mathrm{g} \mathrm{ml}^{-1} \mathrm{~h}^{-1}$ ) during exponentialphase growth (Fig. 4). When growth decelerated due to self-shading of filaments, glycogen synthesis ceased. Within $33 \mathrm{~h}$, the heterocyst-free culture had completely consumed the ammonia which was present in the culture medium. Thereafter Chl synthesis ceased, while dry weight continued to increase due to photosynthetic activity (Fig. 3). Excess products of photosynthesis were stored by glycogen synthesis (Fig. 4). The initial rate of glycogen build-up after $\mathrm{N}$-depletion was $47 \mu \mathrm{g} \mathrm{ml}^{-1} \mathrm{~h}^{-1}$.

On a dry weight basis an initial phase of glycogen accumulation was observed up to $9 \mathrm{~h}$ after inoculation when grown in ammonia. This accumulation compared well with that of a $\mathrm{N}_{2}$-fixing culture under similar growth conditions (Fig. 5, compare also Fig. 2). However, whilst the glycogen content of the $\mathrm{N}_{2}$-fixing culture continuously decreased after having passed the maximum, a second phase of glycogen accumulation was observed in the heterocyst-free culture 


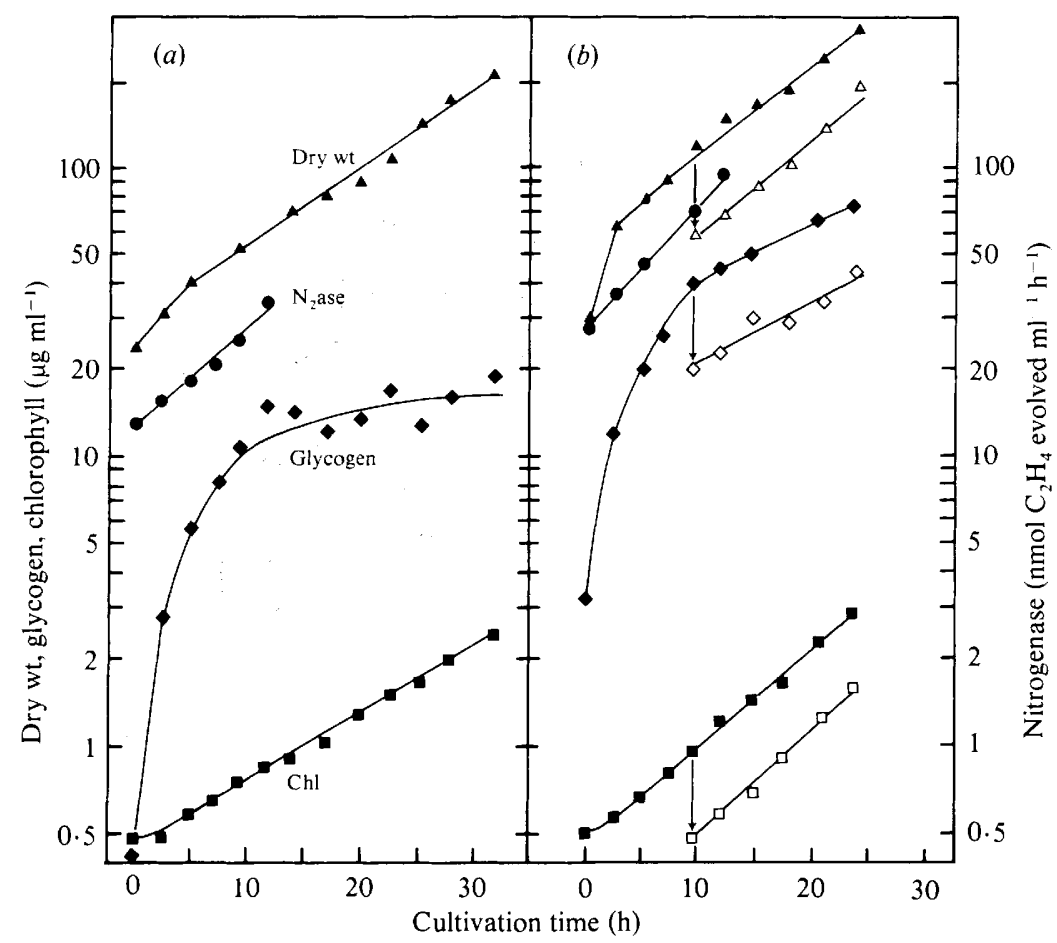

Fig. 1. Exponential-phase growth at two light intensities of $\mathrm{N}_{2}$-fixing filaments of $A$. variabilis. Filaments were grown in mineral medium without combined nitrogen, and were illuminated with fluorescent white light at $4 \mathrm{~W} \mathrm{~m}^{-2}(a)$ and $12 \mathrm{~W} \mathrm{~m}^{-2}(b)$. Dry weight $(\boldsymbol{\Delta}), \mathrm{Chl}(\boldsymbol{\square})$, glycogen $(\bullet)$ and nitrogenase activity $(\mathcal{O})$ were determined as described in Methods. The arrows in (b) indicate $1: 1$ dilutions of 9-h-old cell suspensions at otherwise constant growth conditions. Note the logarithmic scale of the ordinate.

following the cessation of ammonia availability (Fig. 5). The maximum cellular glycogen concentration ( $33 \%$ of dry weight) was attained within $9 \mathrm{~h}$ of the dilution of the culture and within $20 \mathrm{~h}$ of the depletion of ammonia. The initial rates of glycogen buildup on a dry weight basis were $45 \mu \mathrm{g}(\mathrm{mg} \text { dry wt })^{-1} \mathrm{~h}^{-1}$ at $t=0$ and $35 \mu \mathrm{g}(\mathrm{mg} \text { dry wt })^{-1} \mathrm{~h}^{-1}$ at $t=36 \mathrm{~h}$ of growth. The minimum rate of photosynthesis necessary to support this glycogen accumulation can be calculated to be $170 \mu \mathrm{mol} \mathrm{O}_{2}(\mathrm{mg} \mathrm{Chl})^{-1} \mathrm{~h}^{-1}$ and $120 \mu \mathrm{mol} \mathrm{O}_{2}(\mathrm{mg} \mathrm{Chl})^{-1} \mathrm{~h}^{-1}$ respectively. This may indicate a reduced photosynthetic activity $36 \mathrm{~h}$ after inoculation, which is also evident from a decelerated increase in dry weight at this stage of growth (Fig. 3).

Heterocysts were formed and nitrogenase activity rapidly increased $10 \mathrm{~h}$ after ammonia was exhausted (see Bradley \& Carr, 1976, for a detailed description). This allowed further Chl synthesis at about $15 \mathrm{~h}$ after ammonia-depletion (Fig. 3). Little increase in dry weight (Fig. 3) and a net degradation of glycogen (Fig. 4) indicate strong light-limitation of photosynthetic activity $45 \mathrm{~h}$ after inoculation.

\section{Chlorophyll content of cells}

Due to the large variation in cellular glycogen content, the Chl-fraction of the dry weight also varied. As growth is defined as a balanced increase of all cell constituents, accumulation of storage material does not represent growth. It was therefore suggested that storage products should be subtracted from dry weight when referring to growth (van Liere et al., 1979). Fig. 6 shows that although subtracting glycogen from dry weight damped the variations in the Chl- 


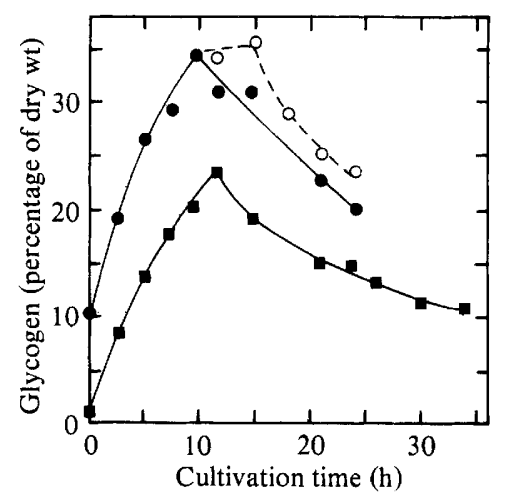

Fig. 2

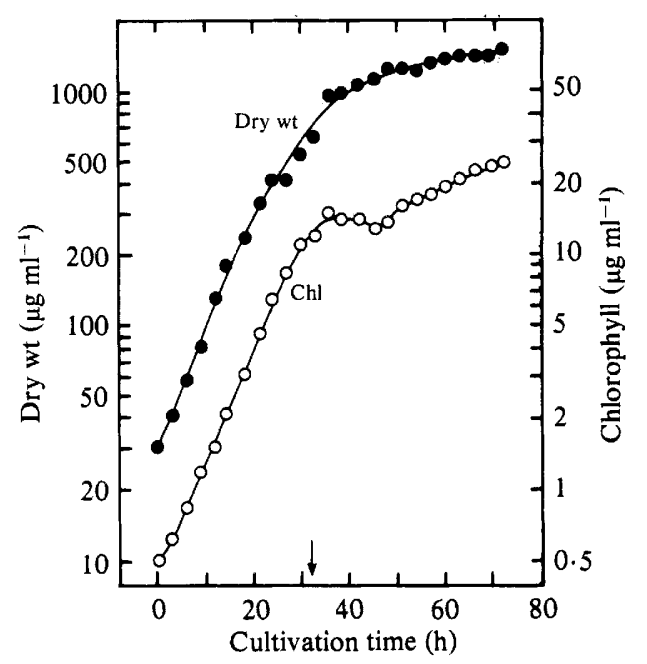

Fig. 3

Fig. 2. Glycogen content of $\mathrm{N}_{2}$-fixing filaments of $A$. variabilis, grown at two light intensities. The glycogen content of filaments grown at $4 \mathrm{~W} \mathrm{~m}^{-2}(\boldsymbol{\square})$ or $12 \mathrm{~W} \mathrm{~m}^{-2}(\mathbf{O})$, as shown in Fig. $1(a)$ and $1(b)$, respectively, was calculated as a percentage $(w / w)$ of the dry weight. $O$, Cellular glycogen content of a culture suspension diluted $(1: 1)$ after $9 \mathrm{~h}$ growth at $12 \mathrm{~W} \mathrm{~m}^{-2}$ (compare Fig. $1 b$ ).

Fig. 3. Growth of a heterocyst-free $A$. variabilis culture on a limited amount of ammonia. To obtain heterocyst-free filaments, $A$. variabilis was precultivated in a mineral medium containing ammonia ( $6 \mathrm{~mm}$ initial concentration, renewed daily) at $12 \mathrm{~W} \mathrm{~m}^{-2} .0$, Dry weight; $O, \mathrm{Chl}$. The arrow indicates the cessation of ammonium supply in the mineral medium, when the medium was not changed after $24 \mathrm{~h}$.

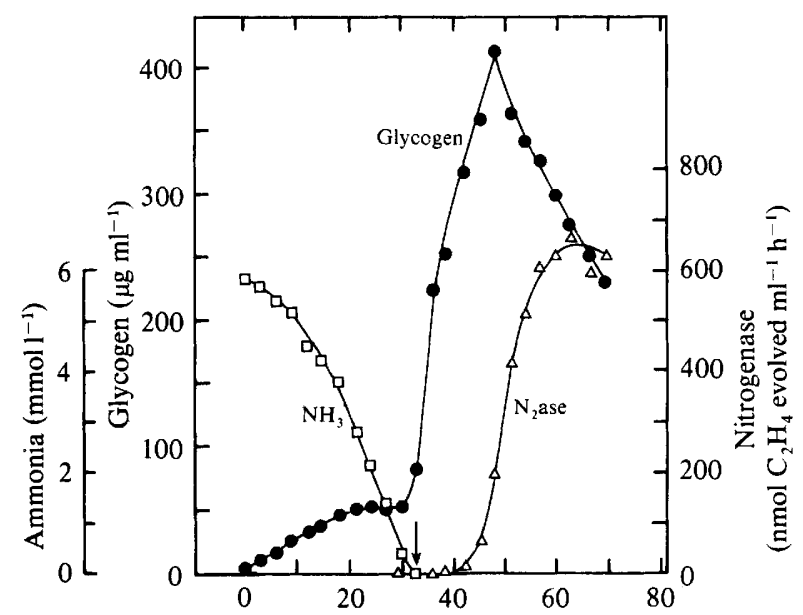

Fig. 4. Net glycogen synthesis, ammonia-depletion, and induction of nitrogenase activity in $A$. variabilis. A culture of $A$, variabilis was precultivated to become heterocyst-free and grown as indicated in Fig. 3. $\square$, Ammonium consumption; , glycogen synthesis; $\triangle$, nitrogenase activity. Note the linear scale of the ordinate.

fraction, $\mathrm{Chl}$ still did not represent a constant cellular value (Fig. 6). As the average light intensity available for the filaments changed markedly during batch cultivation, specific changes in the pigmentation (Foy \& Gibson, 1982) may contribute to the residual variation and deserve further attention. A correction of growth rates based on $\mathrm{Chl}$ was not done. 


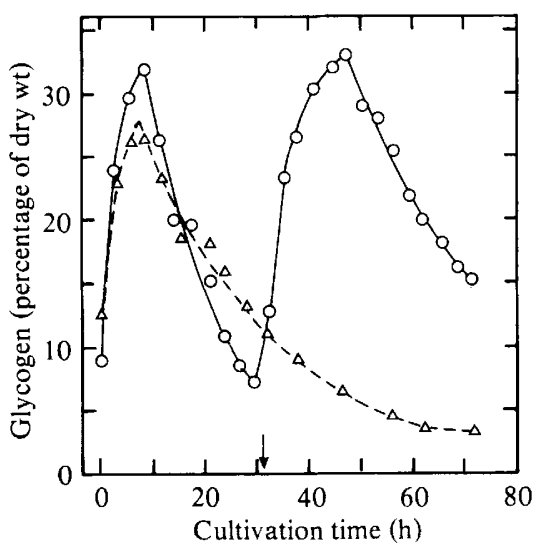

Fig. 5. Glycogen content of $A$. variabilis grown on a limited amount of ammonia or with $\mathrm{N}_{2}$ as the sole nitrogen source. Glycogen content was calculated as a percentage $(w / w)$ of the dry weight. $\triangle$, Glycogen content of a $\mathrm{N}_{2}$-fixing culture; $\mathrm{O}$, glycogen content of an ammonia-assimilating culture, which initially was free of heterocysts and nitrogenase activity. The arrow indicates the cessation of ammonia supply to the heterocyst-free culture (compare also Figs 3 and 4).

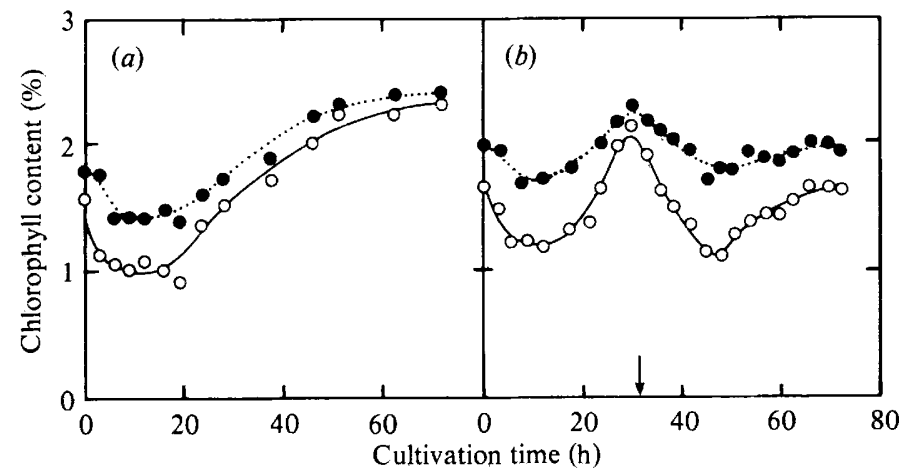

Fig. 6. Chl content of $A$. variabilis as related to total dry weight and dry weight minus glycogen. Chl content is given as a percentage $(w / w)$ of dry weight $(O)$ and as a percentage of the remaining cell material () which was calculated by subtracting the weight of glycogen from the dry weight as suggested by van Liere et al., 1979. (a) $\mathrm{N}_{2}$-fixing culture; (b) heterocyst-free culture (the arrow indicates the cessation of the ammonia supply).

\section{DISCUSSION}

In continuously illuminated cultures of cyanobacteria, glycogen synthesis and degradation occur simultaneously (Lehmann \& Wöber, 1976). A net accumulation or degradation might be the result of a shift in the activity of one or both processes due to changing growth conditions. Characteristics of isolated ADP-glucose pyrophosphorylase indicate a regulation of glycogen synthesis by 3-phosphoglycerate and orthophosphate in cyanobacteria as well as in plants (Levi \& Preiss, 1976). This would allow photosynthesis to influence glycogen synthesis directly.

During batch cultivation, a growth cycle passes a series of phases from inoculation to an eventual cessation of growth, due to a continuous change in light irradiance caused by mutual shading during the increase in biomass. In our experiments, we assumed an increase in the average irradiation of filaments upon dilution. This increase not only stimulated exponential growth but also caused the photosynthetically active cells to accumulate glycogen. Growth rate, 
rate of synthesis and maximum storage capacity increased with increasing light intensity (Figs 1 and 2). This is in marked contrast to most reports on glycogen accumulation in bacteria, where rate of growth and the quantity of glycogen accumulated exhibit an inverse relationship (Preiss, 1984).

Glycogen was also accumulated in young, non-differentiated batch cultures grown in the presence of ammonia (Fig. 5). Upon exhaustion of ammonia, Chl synthesis stopped while dry weight continued to increase due to photosynthesis. In this situation, glycogen acted to buffer photosynthetic activity until the induction of nitrogenase allowed for further growth. In $\mathrm{N}_{2}$ fixing cultures, the initial glycogen accumulation was accompanied by a small rise in nitrogenase activity when compared to $\mathrm{Chl}$ or dry weight (see also Ernst et al., 1984). A Nlimitation induced by the acceleration of growth upon dilution can, therefore, be proposed. Induction of heterocysts or nitrogenase activity was, however, not necessarily involved in glycogen accumulation, as shown by its absence during glycogen accumulation in nondifferentiated filaments (Fig. 5, first accumulation).

The accumulation of glycogen in the cells upon dilution resembled the glycogen synthesis of cultures grown in diurnal cycles as characterized by a linear increase, despite the exponential growth of the culture, and by the maximum cellular glycogen concentration being correlated with light intensity (van Liere et al., 1979; Loogmann, 1982). We have additionally shown that accumulation can occur irrespective of the $\mathrm{N}$-source and, as long as the $\mathrm{N}$-supply is guaranteed, without heterocyst differentiation. This can be considered to be a useful capacity in a phototroph, which uses glycogen to overcome periodic interruptions of energy supply during darkness, without changing the state of other metabolic reactions.

The $\mathrm{C}: \mathrm{N}$ ratio of cyanobacterial cells has been suggested to signal the differentiation of heterocysts (Kulasooriya et al., 1972; Bradley \& Carr, 1976). On a molecular basis such signals may be represented by metabolite or effector ratios or levels but their identity is still unknown (see Wolk, 1982, for further discussion). As our results show glycogen accumulation occurring independently of differentiation, a change in $\mathrm{C}: \mathrm{N}$ ratio by whatever metabolite is unlikely to regulate heterocyst and nitrogenase formation. Further investigations using clearly defined conditions may not only help to explain the controversy in the assessment of glycogen buildup in phototrophic and heterotrophic bacteria (Mur, 1983), but may also help to identify signals for heterocyst differentiation.

This study was part of a project within the Zentrum für Energieforschung of this University.

\section{REFERENCES}

Allen, M. M. (1984). Cyanobacterial cell inclusions. Annual Review of Microbiology 38, 1-25.

Allen, M. M. \& Smith, A. J. (1969). Nitrogen chlorosis in blue-green algae. Archiv für Mikrobiologie 69, 114-120.

ARnon, D. I., MCSwain, B. D., Tsujimoto, H. T. \& WADA, K. (1974). Photochemical activity and components of membrane preparation from bluegreen algae. I. Biochimica et biophysica acta 357, 231 245.

Bradley, S. \& CarR, N. G. (1976). Heterocysts and nitrogenase development in Anabaena cylindrica. Journal of General Microbiology 96, 175-184.

Chaney, A. L. \& Marbach, E. P. (1962). Modified reagents for the determination of urea and ammonia. Clinical Chemistry 8, 130-132.

EIDELs, L. \& PREISS, J. (1970). Carbohydrate metabolism in Rhodopseudomonas capsulata. Archives of Biochemistry and Biophysics 140, 75-89.

ERnSt, A., Kirschenlohr, H., Diez, J. \& Böger, P. (1984). Glycogen content and the stability of nitrogenase activity in Anabaena variabilis. Archives of Microbiology 140, 120-125.
VAN EyKelenburg, C. (1980). Ecophysiological studies on Spirulina platensis: effect of temperature, light intensity and nitrate concentration on growth and ultrastructure. Antonie van Leeuwenhoek 46, 113127.

Foy, R. H. \& Gibson, C. E. (1982). Photosynthetic characteristics of planktonic blue-green algae: changes in photosynthetic capacity and pigmentation of Oscillatoria redekei van Goor under high and low light. British Phycological Journal 17, 183193.

Kromkamp, J. \& MUR, L. R. (1984). Buoyant density changes in the cyanobacterium Microcystis aeruginosa due to changes in the cellular carbohydrate content. FEMS Microbiology Letters 25, 105-109.

Kulasooriya, S. A., LANG, N. J. \& Fay, P. (1972). The heterocysts of blue green algae. III. Differentation and nitrogenase activity. Proceedings of the Royal Society B181, 199-209.

LehmanN, M. \& WöBER, G. (1976). Accumulation, mobilisation and turn-over of glycogen in the bluegreen bacterium Anacystis nidulans. Archives of Microbiology 111, 93-97. 
LeVI, C. \& Preiss. J. (1976). Regulatory properties of the ADP-glucose pyrophosphorylase of the blue green bacterium Syechococcus 6301. Plant Physio$\log v$ 58, 753-756.

van Liere, L., Mur, L. R., Gibson, C. E. \& Herdman, M. (1979). Growth and physiology of Oscillatoria agardhii Gomont cultivated in continuous cultures with a light-dark cycle. Archices of Microhiology 123 , 315-318.

LoOGMAN, J. G. (1982). Influence of photoperiodicity on algal growth kinetics. $\mathrm{PhD}$ thesis, University of Amsterdam.

MUR, L. R. (1983). Some aspects of the ecophysiology of cyanobacteria. Annales de microbiologie 134B, 61 72

Preiss, J. (1984). Bacterial glycogen synthesis and its regulation. Annual Review of Microbiology 38, 40--58.
Rippka, R. \& Stanier, R. Y. (1978). The effects of anaerobiosis on nitrogenase synthesis and heterocyst development by nostocacean cyanobacteria. Journal of General Microbiology 105, 83-94.

SMITH, A. J. (1982). Modes of cyanobacterial carbon metabolism. In The Biology of Cyanobacteria, pp. 47 86. Edited by N. G. Carr \& B. A. Whitton. Oxford: Blackwell Scientific Publications.

DE VASConcelos, L. \& FAY, P. (1974). Nitrogen metabolism and ultrastructure in Anabaena cylindrica. I. Archives of Microbiology 96, 271-279.

WOLK, C. P. (1982). Heterocysts. In The Biology of Cyanobacteria, pp. 359-387. Edited by N. G. Carr \& B. A. Whitton. Oxford: Blackwell Scientific Publications. 